TRANSACTIONS OF THE

AMERICAN MATHEMATICAL SOCIETY

Volume 361, Number 12, December 2009, Pages 6429-6446

S 0002-9947(09)04793-X

Article electronically published on June 17, 2009

\title{
A CLASS OF LOCAL CLASSICAL SOLUTIONS FOR THE ONE-DIMENSIONAL PERONA-MALIK EQUATION
}

\author{
MARINA GHISI AND MASSIMO GOBBINO
}

Abstract. We consider the Cauchy problem for the one-dimensional PeronaMalik equation

$$
u_{t}=\frac{1-u_{x}^{2}}{\left(1+u_{x}^{2}\right)^{2}} u_{x x}
$$

in the interval $[-1,1]$, with homogeneous Neumann boundary conditions.

We prove that the set of initial data for which this equation has a localin-time classical solution $u:[-1,1] \times[0, T] \rightarrow \mathbb{R}$ is dense in $C^{1}([-1,1])$. Here "classical solution" means that $u, u_{t}, u_{x}$ and $u_{x x}$ are continuous functions in $[-1,1] \times[0, T]$.

\section{INTRODUCTION}

In this paper we consider the initial boundary value problem

$$
\begin{array}{ll}
u_{t}=\frac{1-u_{x}^{2}}{\left(1+u_{x}^{2}\right)^{2}} u_{x x} & \text { in }[-1,1] \times[0, T], \\
u_{x}(-1, t)=u_{x}(1, t)=0 & \forall t \in[0, T], \\
u(x, 0)=u_{0}(x) & \forall x \in[-1,1] .
\end{array}
$$

Since we are interested in classical solutions, we require that (1.1) and (1.2) be satisfied also for $t=0$. In particular we always consider initial conditions $u_{0} \in C^{2}([-1,1])$ and such that $u_{0 x}(-1)=u_{0 x}(1)=0$.

Formally, equation (1.1) is an instance of the usual parabolic PDE in divergence form $u_{t}=\left(\varphi^{\prime}\left(u_{x}\right)\right)_{x}=\varphi^{\prime \prime}\left(u_{x}\right) u_{x x}$, corresponding to $\varphi(\sigma)=2^{-1} \log \left(1+\sigma^{2}\right)$. The main feature is that $\varphi(\sigma)$ is convex for $|\sigma|<1$ and concave for $|\sigma|>1$. This implies that (1.1) is a forward-parabolic PDE where $\left|u_{x}\right|<1$ (the forward or subcritical region) and a backward-parabolic PDE where $\left|u_{x}\right|>1$ (the backward or supercritical region).

Equation (1.1) is the one-dimensional version of the diffusion process introduced in 15 in the context of image processing. Numerical computations seem to show that this equation produces the desired denoising effect on the initial condition $u_{0}$ despite the expected ill posedness: this is usually referred to as "the Perona-Malik paradox".

Mathematical understanding of this phenomenology is still quite poor. Research has developed along three main lines: finding a suitable notion of weak solution

Received by the editors November 13, 2006 and, in revised form, October 25, 2007.

2000 Mathematics Subject Classification. Primary 35A07, 35B65, 35K65.

Key words and phrases. Perona-Malik equation, classical solution, forward-backward parabolic equation, anisotropic diffusion, supersolutions, comparison principles.

(C)2009 American Mathematical Society Reverts to public domain 28 years from publication 
consistent with experiments [4, 7, 9, 13, 16, 17, proving a priori estimates on classical solutions yielding existence or nonexistence results [8, 9, 12, extending some one-dimensional results to higher dimension [5, 9.

In this paper we focus on classical solutions, namely solutions with one derivative with respect to time and two derivatives in the space variable. The following results are by now well established in the literature.

- If $u_{0}(x)$ is subcritical, i.e., $\left|u_{0 x}(x)\right|<1$ for all $x \in[-1,1]$, then problem(1.1), (1.2), (1.3) has a global (defined for every $t \geq 0$ ) classical solution which remains subcritical for all times [12].

- If $u_{0}(x)$ is transcritical, i.e., $\varphi^{\prime \prime}\left(u_{0 x}\right)$ changes its sign in $[-1,1]$, then classical solutions, if they exist, cannot be global [12, 10. In particular in [10] it is proved that for a transcritical solution we have that necessarily

$$
T \leq 4 \int_{-1}^{1} \log \left(1+u_{0 x}^{2}(x)\right) d x .
$$

- Let us assume that a classical local solution exists for some transcritical $u_{0}(x)$, and let us consider any closed interval contained in the supercritical region of $u_{0}$. As remarked in [13, in this interval $u_{0}$ is the trace at $t=0$ of the solution of a backward strictly parabolic equation. Therefore the standard regularity theory provides severe restrictions on $u_{0}$. For example, a necessary (but by no means sufficient) condition is that $u_{0}$ is of class $C^{\infty}$ in its supercritical region.

To our knowledge, up to now no example of local classical solution with a transcritical $u_{0}$ had been shown. We refer the reader to [12, Section 6] for a discussion on some apparently inconclusive approaches to local solutions, including higher order regularization, vanishing viscosity, power series expansions.

Some signs even made people skeptical about their existence. From the analytical point of view it was proved in [10] that the trivial stationary solutions $u(x, t)=a x+$ $b$ are the unique classical solutions of (1.1) defined for every $(x, t) \in \mathbb{R}^{2}$. From the numerical point of view, experiments on some regularizations of (1.1) showed a rapid formation of microstructures with a drastic reduction of the energy in the backward region 3]. This phenomenon, usually referred to as staircasing [13 or fibrillation [6], happens in a time scale which vanishes with the regularization parameter 3 . After the formation of microstructures the evolution in the backward region slows down and the dynamic is governed by the forward region. This may lead one to suspect that in the limit the evolution of any transcritical initial condition should exhibit the instantaneous formation of discontinuities in $u_{x}$ and maybe also in $u$.

We show that this is not always the case, because some local classical transcritical solutions do exist. We have indeed the following existence result (throughout this paper $C^{2,1}$ denotes the usual parabolic space of functions $u(x, t)$ such that $u, u_{t}$, $u_{x}$ and $u_{x x}$ are continuous functions).

Theorem 1.1. Let $\mathcal{R} \subseteq C^{1}([-1,1])$ be the set of initial data $u_{0}(x)$ for which there exist $T>0$ and $u \in C^{2,1}([-1,1] \times[0, T])$ satisfying (1.1), (1.2), and (1.3).

Then $\mathcal{R}$ is dense in $C^{1}([-1,1])$.

The same is true for all equations $u_{t}=\varphi^{\prime \prime}\left(u_{x}\right) u_{x x}$ with $\varphi \in C^{\infty}(\mathbb{R})$ (this regularity requirement can probably be weakened) and such that the set $\{\sigma \in \mathbb{R}$ : $\left.\varphi^{\prime \prime}(\sigma)=0\right\}$ has no accumulation points. 
Note that in Theorem 1.1, in order to obtain density, we are forced to admit that the life span $T$ of the solution depends on the initial condition. This may seem too restrictive, but it is not. Indeed there are transcritical data for which the right hand side of (1.4) is arbitrarily small: it follows that the set of initial data for which a classical solution exists at least on a fixed time interval $[0, T]$ cannot be dense in $C^{1}([-1,1])$.

Our proof does not characterize all initial data for which a local solution exists (and we suspect that a nontautological characterization does not exist). We only exhibit a quite special class of such data, which however turns out to be dense in $C^{1}([-1,1])$, hence a fortiori in $C^{0}([-1,1])$ or in $L^{2}((-1,1))$. On the other hand 13 shows that nothing more than density can be expected.

The rough idea of our construction is the following. Let us take any $v_{0} \in$ $C^{\infty}([-1,1])$ such that $\left|v_{0 x}(x)\right|=1$ in a finite number of points. Let $I^{+}$and $I^{-}$be the subcritical and the supercritical regions of $v_{0}$, respectively (both $I^{+}$and $I^{-}$are finite unions of intervals). Given $T>0$, we prove (Theorem 2.1) that there exists a solution $u$ of (1.1), (1.2) which at $t=0$ coincides with $v_{0}$ in $I^{+}$and at $t=T$ coincides with $v_{0}$ in $I^{-}$. This means that $v_{0}$ acts as an initial condition in $I^{+}$and as a final condition in $I^{-}$. In order to construct such a solution, we solve a separate problem in any connected component of $I^{+}$and $I^{-}$: this subproblem is always well posed because it is either a (degenerate) forward parabolic problem with an initial condition or a (degenerate) backward parabolic problem with a final condition. The main (somehow unexpected) point is that the solutions of these subproblems glue together in a $C^{2,1}$ way provided that $T$ is small enough and $v_{0 x x}(x)=0$ whenever $\left|v_{0 x}(x)\right|=1$. The required estimates are proved in Theorem 2.2 and Theorem 2.3 using standard energy estimates in the interior and suitable subsolutions and supersolutions to control the behavior near the critical points where $\left|v_{0 x}(x)\right|=1$. More precisely, the key steps are the paragraph Uniform vanishing of $u_{x x}^{\varepsilon}$ at the boundary in the proof of Theorem 2.3 and the paragraph Existence of $u_{x}$ at the boundary in the proof of Theorem 2.2 .

If we now define $u_{0}(x)$ as $u(x, 0)$, we have found an initial condition for which (1.1), (1.2), (1.3) has a local solution; hence the set $\mathcal{R}$ is nonempty. Notice that $u_{0}$ coincides with $v_{0}$ in $I^{+}$, while in $I^{-}$it is the trace at $t=0$ of a solution of a backward problem, as required by [13].

Finally, if we choose $T$ small enough, it is clear that $u_{0}$ is as close to $v_{0}$ as we want in the $C^{2}([-1,1])$ topology. Since the set of admissible $v_{0}$ (see Theorem 2.1) is in turn dense in $C^{1}([-1,1])$, we easily conclude that $\mathcal{R}$ is dense in $C^{1}([-1,1])$.

We conclude by speculating on some consequences of Theorem 1.1

From the analytical point of view, classical solutions are naturally welcome. They also give a new light to a priori estimates on smooth solutions 9, 12. Up to now indeed it was suspected that they were likely to be vacuous in the transcritical case.

From the numerical point of view things may be different. On one hand classical solutions are not a solution of the Perona-Malik paradox, since there is no reason for them to be stable or to exist but for special classes of smooth initial data (while a good theory is expected for $B V$ data). On the other hand, it is easy to see that classical solutions must be taken into account by any reasonable stable theory. We state it formally in the case of the fourth order regularization (Remark 2.4), but analogous statements are true for the semi-discrete scheme and maybe also 
for several other approximations of (1.1). The rough idea is the following: our classical solutions can be obtained as limits in the $C^{2,1}$ topology of solutions of the regularized problems up to adding a small perturbation which vanishes in the $C^{0}$ norm with the regularization parameter.

This proves that any stable theory allowing perturbations vanishing in $C^{0}$ and convergence of solutions in $C^{2,1}$ cannot ignore the dynamics in supercritical regions, as to the contrary it seemed to be suggested by numerical experiments and some analytical results on simpler models [4. For this reason, a solution of the PeronaMalik paradox now seems even further away.

Remark 2.4 can of course be compared with [9, where it is shown that any theory allowing perturbations vanishing in $L^{2}$ and uniform convergence of solutions contains the stationary solution for every initial datum in $B V$, and with [16] (see also the pioneering paper [11) where it is shown that any theory allowing perturbations vanishing in $W^{-1, \infty}$ and uniform convergence of solutions contains plenty of exotic solutions even for smooth subcritical data.

The plan of this paper is the following. In section 2 we state Theorem 2.1 and we show how its proof reduces to the proof of Theorem 2.2 and Theorem 2.3 . In section 3 we give the details of the proofs.

\section{Statements}

As we have seen in the introduction, Theorem 1.1 is a straightforward consequence of the following result, where we prove well posedness for (1.1), (1.2) with a mixture of initial and final conditions.

Theorem 2.1. Let $n \in \mathbb{N}$, and let $-1=a_{0}<a_{1}<\ldots<a_{2 n}<a_{2 n+1}=1$. Let us consider the open sets

$$
I^{-}:=\bigcup_{i=1}^{n}\left(a_{2 i-1}, a_{2 i}\right), \quad I^{+}:=\bigcup_{i=0}^{n}\left(a_{2 i}, a_{2 i+1}\right) .
$$

Let $v_{0} \in C^{\infty}([-1,1])$ be a function such that

(i) $\left|v_{0 x}(x)\right|<1$ for every $x \in I^{+}$and $\left|v_{0 x}(x)\right|>1$ for every $x \in I^{-}$;

(ii) $v_{0 x x}(x)=0$ for every $x \in\left\{a_{1}, \ldots, a_{2 n}\right\}$;

(iii) all derivatives of $v_{0}$ with odd order are zero at $x=-1$ and $x=1$.

Then for every $T>0$ small enough there exists $u \in C^{2,1}([-1,1] \times[0, T])$ satisfying (1.1), (1.2), and

$$
\begin{aligned}
u(x, 0) & =v_{0}(x) & & \forall x \in I^{+}, \\
u(x, T) & =v_{0}(x) & & \forall x \in I^{-}, \\
u(x, t) & =v_{0}(x) & & \forall(x, t) \in\left\{a_{1}, \ldots, a_{2 n}\right\} \times[0, T] .
\end{aligned}
$$

Moreover

$$
\begin{gathered}
\left|u_{x}(x, t)\right|<1 \text { in } I^{+} \times[0, T] \text { and }\left|u_{x}(x, t)\right|>1 \text { in } I^{-} \times[0, T], \\
u_{x}(x, t)=v_{0 x}(x) \quad \forall(x, t) \in\left\{a_{1}, \ldots, a_{2 n}\right\} \times[0, T], \\
u_{x x}(x, t)=0 \quad \forall(x, t) \in\left\{a_{1}, \ldots, a_{2 n}\right\} \times[0, T] .
\end{gathered}
$$

The situation described in Theorem 2.1 is represented in Figure 1 in the particular case $n=2$. The function $v_{0}$ is prescribed as an initial condition in the lower horizontal thick segments, as a final condition in the upper horizontal thick segments, and as a Dirichlet condition in the vertical thick segments. We have 


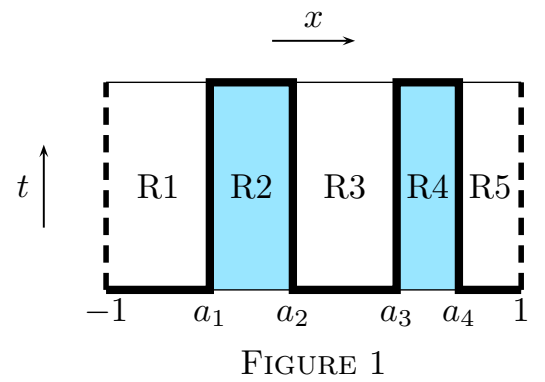

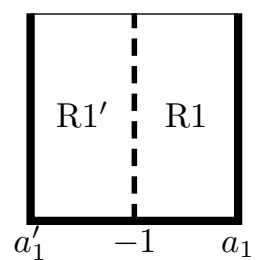

FIGURE 2

Neumann boundary conditions in the vertical dashed segments. The shaded regions represent the supercritical zone $I^{-} \times[0, T]$.

In the general case the rectangle $[-1,1] \times[0, T]$ is divided into $2 n+1$ subrectangles. Now we solve (1.1) separately in each subrectangle with the appropriate boundary conditions. We end up with problems of three types.

- $D$-D-forward problems. In rectangles $\left(a_{2 i}, a_{2 i+1}\right) \times[0, T](i=1, \ldots, n-1)$ (R3 in the figure) we have a forward parabolic equation with an initial datum and Dirichlet boundary conditions.

- $D$-D-backward problems. In rectangles $\left(a_{2 i-1}, a_{2 i}\right) \times[0, T](i=1, \ldots, n)(\mathrm{R} 2$ and R4 in the figure) we have a backward parabolic equation with a final datum and Dirichlet boundary conditions. These problems can be reduced to D-D-forward problems just by reversing the time.

- $N$-D-forward problems. In the lateral rectangles $\left(-1, a_{1}\right)$ $\times[0, T]$ and $\left(a_{2 n}, 1\right) \times[0, T](\mathrm{R} 1$ and $\mathrm{R} 5$ in the figure) we have a forward parabolic equation with an initial datum, a Dirichlet boundary condition in one of the endpoints of the space interval, and a homogeneous Neumann boundary condition in the other endpoint. These problems can be reduced to D-D-forward problems with a standard reflection argument. For example in the case of the rectangle on the left we can define $a_{1}^{\prime}$ as the symmetric of $a_{1}$ with respect to -1 (see Figure 2 ) and extend $v_{0}$ to the interval $\left[a_{1}^{\prime}, a_{1}\right]$ in such a way that $v_{0}(-1-x)=v_{0}(-1+x)$. By assumption (iii) in Theorem 2.1 the extension is still of class $C^{\infty}$.

The solution of the symmetrized problem is clearly symmetric with respect to $x=-1$. It follows that its restriction to $\left[-1, a_{1}\right] \times[0, T]$ satisfies the homogenous Neumann boundary condition at $x=-1$.

It is clear that for every choice of $T>0$ the solutions found in the subrectangles glue together in a $C^{0}$ way. The main point is proving that the glueing is actually of class $C^{2,1}$. This is where the smallness of $T$ and assumption (ii) in Theorem 2.1 play a fundamental role. Under such assumptions indeed it turns out that not only $u(x, t)$ coincides with $v_{0}(x)$ for $x \in\left\{a_{1}, \ldots, a_{2 n}\right\}$, but also $u_{x}(x, t)$ and $u_{x x}(x, t)$ coincide with $v_{0 x}(x)$ and $v_{0 x x}(x)$, respectively, at the same points. In a certain sense conditions (2.2) and (2.3) make the problem overdetermined, but the degeneracy of the equation at the boundary of the subrectangle yields some sort of finite speed of propagation in such a way that $u, u_{x}$ and $u_{x x}$ stay constant at the boundary for some small time.

As we have just seen, in the proof of these results we can limit ourselves to D-D-forward problems. The general setting is the following. We have a function 
$\varphi \in C^{\infty}(\mathbb{R})$ and an interval $(\alpha, \beta)$ such that

$$
\varphi^{\prime \prime}(\sigma)>0 \text { for every } \sigma \in(\alpha, \beta)
$$

Then we have a function $u_{0} \in C^{\infty}([a, b])$ such that

$$
\begin{gathered}
\alpha<u_{0 x}(x)<\beta \quad \forall x \in(a, b), \\
\varphi^{\prime \prime}\left(u_{0 x}(a)\right)=\varphi^{\prime \prime}\left(u_{0 x}(b)\right)=0, \\
u_{0 x x}(a)=u_{0 x x}(b)=0 .
\end{gathered}
$$

We finally consider the degenerate parabolic problem

$$
\begin{array}{ll}
u_{t}=\varphi^{\prime \prime}\left(u_{x}\right) u_{x x} & \text { in }[a, b] \times[0,+\infty), \\
u(x, t)=u_{0}(x) & \forall(x, t) \in \partial_{P}([a, b] \times[0,+\infty)),
\end{array}
$$

where $\partial_{P}$ denotes the parabolic boundary. We recall that the parabolic boundary of a rectangle $[a, b] \times[0, T)$ (with possibly $T=+\infty$ ) or $[a, b] \times[0, T]$ is the set of its points with either $t=0$ or $x \in\{a, b\}$.

Therefore in (2.10) the function $u_{0}$ gives both the initial condition at $t=0$ and the Dirichlet boundary condition at the endpoints $x=a$ and $x=b$.

All the problems in the subrectangles fit in this framework. In the D-D-forward problems and in the N-D-forward problems after reflection we can take $\varphi(\sigma)=$ $2^{-1} \log \left(1+\sigma^{2}\right)$ and $(\alpha, \beta)=(-1,1)$. As for D-D-backward problems, after reversing the time, we may assume $\varphi(\sigma)=-2^{-1} \log \left(1+\sigma^{2}\right)$ and $(\alpha, \beta)$ defined as follows: if $u_{0 x}>1$ in the given subinterval, then we take $\alpha=1$ and $\beta$ any strict upper bound for $u_{0 x}$; if $u_{0 x}<-1$ in the given subinterval, then we take $\beta=-1$ and $\alpha$ any strict lower bound for $u_{0 x}$. In all the cases $u_{0}$ is either $v_{0}$ or its extension by reflection in the N-D-forward problems.

Theorem 2.2. Let us assume that $\varphi$ and $u_{0}$ satisfy (2.4) through (2.8).

Then there exists a unique function $u \in C^{0}([a, b] \times[0,+\infty)) \cap C^{\infty}((a, b) \times[0,+\infty))$ satisfying (2.9), (2.10), and the estimate

$$
\alpha<u_{x}(x, t)<\beta \quad \forall(x, t) \in(a, b) \times[0,+\infty) .
$$

Moreover there exists $T_{0}>0$ such that $u \in C^{2,1}\left([a, b] \times\left[0, T_{0}\right]\right)$ and

$$
\begin{gathered}
u_{x}(a, t)=u_{0 x}(a), u_{x}(b, t)=u_{0 x}(b) \quad \forall t \in\left[0, T_{0}\right], \\
u_{x x}(a, t)=u_{x x}(b, t)=0 \quad \forall t \in\left[0, T_{0}\right] .
\end{gathered}
$$

Some parts of Theorem 2.2 are probably scattered in the huge porous medium literature. The $C^{2,1}$ regularity is in any case beyond the optimal regularity for this type of equations [1, 2], and this is of course due to assumptions (2.7) and (2.8). In order to take advantage of these assumptions, we give a self-contained proof of Theorem 2.2, which only relies on the theory of strictly parabolic problems [14].

To this end, given $\varepsilon>0$, we approximate $u_{0}(x)$ with

$$
u_{0}^{\varepsilon}(x):=\frac{u_{0}(x)}{1+\varepsilon}+\frac{\varepsilon(\alpha+\beta)}{2(1+\varepsilon)} x .
$$

The motivation of this choice is that if $\alpha \leq u_{0 x}(x) \leq \beta$ for every $x \in[a, b]$, then there exist constants $\alpha_{\varepsilon}$ and $\beta_{\varepsilon}$ such that

$$
\alpha<\alpha_{\varepsilon} \leq u_{0 x}^{\varepsilon}(x) \leq \beta_{\varepsilon}<\beta \quad \forall x \in[a, b] .
$$


Now we consider the problem

$$
\begin{array}{ll}
u_{t}^{\varepsilon}=\varphi^{\prime \prime}\left(u_{x}^{\varepsilon}\right) u_{x x}^{\varepsilon} & \text { in }[a, b] \times[0,+\infty), \\
u^{\varepsilon}(x, t)=u_{0}^{\varepsilon}(x) & \forall(x, t) \in \partial_{P}([a, b] \times[0,+\infty)) .
\end{array}
$$

This problem turns out to be strictly parabolic. The following result collects the estimates that are needed for the proof of Theorem 2.2

Theorem 2.3. Let us assume that $\varphi$ and $u_{0}$ satisfy (2.4) through (2.8). Let $\varepsilon>0$, and let $u_{0}^{\varepsilon}, \alpha_{\varepsilon}$ and $\beta_{\varepsilon}$ be defined by (2.14) and (2.15).

Then there exists a unique function

$$
u^{\varepsilon} \in C^{2,1}([a, b] \times[0,+\infty)) \cap C^{\infty}((a, b) \times[0,+\infty)) \cap C^{\infty}([a, b] \times(0,+\infty))
$$

satisfying (2.16), (2.17), and

$$
\begin{array}{cr}
\alpha_{\varepsilon} \leq u_{x}^{\varepsilon}(x, t) \leq \beta_{\varepsilon} & \forall(x, t) \in(a, b) \times[0,+\infty), \\
u_{x x}^{\varepsilon}(x, t)=0 & \forall(x, t) \in\{a, b\} \times[0,+\infty) .
\end{array}
$$

Moreover $u^{\varepsilon}$ satisfies the following estimates independent of $\varepsilon$.

(1) Global estimates on $u_{t}$. There exists a constant $M_{1}$ such that

$$
\begin{gathered}
\int_{a}^{b}\left[u_{t}^{\varepsilon}(x, t)\right]^{2} d x \leq M_{1} \quad \forall t \geq 0, \\
\int_{0}^{T} \int_{a}^{b} \varphi^{\prime \prime}\left(u_{x}^{\varepsilon}(x, t)\right)\left[u_{x t}^{\varepsilon}(x, t)\right]^{2} d x d t \leq M_{1} \quad \forall T \geq 0 .
\end{gathered}
$$

(2) Uniform strict parabolicity in the interior. For every closed interval $\left[x_{1}, x_{2}\right]$ $\subseteq(a, b)$ and every $T>0$ there exist constants $M_{2}$ and $M_{3}$ such that

$$
\alpha<M_{2} \leq u_{x}^{\varepsilon}(x, t) \leq M_{3}<\beta \quad \forall(x, t) \in\left[x_{1}, x_{2}\right] \times[0, T] .
$$

As a consequence, there exists a constant $M_{4}>0$ such that

$$
\varphi^{\prime \prime}\left(u_{x}^{\varepsilon}(x, t)\right) \geq M_{4} \quad \forall(x, t) \in\left[x_{1}, x_{2}\right] \times[0, T] .
$$

(3) Interior estimates. For every closed interval $\left[x_{1}, x_{2}\right] \subseteq(a, b)$ and every $T>0$ there exists a constant $M_{5}$ such that

$$
\begin{aligned}
\int_{x_{1}}^{x_{2}}\left[u_{x t}^{\varepsilon}(x, t)\right]^{2} d x & \leq M_{5} \quad \forall t \in[0, T], \\
\int_{0}^{T} \int_{x_{1}}^{x_{2}}\left[u_{x x t}^{\varepsilon}(x, t)\right]^{2} d x d t & \leq M_{5}, \\
\left|u_{x x}^{\varepsilon}(x, t)\right| & \leq M_{5} \quad \forall(x, t) \in\left[x_{1}, x_{2}\right] \times[0, T] .
\end{aligned}
$$

(4) Uniform vanishing of $u_{x x}^{\varepsilon}$ at the boundary. There exist constants $T_{0}>0$ and $M_{6}$ such that

$$
\left|u_{x x}^{\varepsilon}(x, t)\right| \leq M_{6}(x-a)(b-x) \quad \forall(x, t) \in[a, b] \times\left[0, T_{0}\right] .
$$

We conclude by remarking that classical solutions cannot be neglected when looking for a stable notion of weak solution. We show indeed that they are obtained by fourth order regularization with a vanishing continuous perturbation. 
Remark 2.4. Let $u \in C^{2,1}([-1,1] \times[0, T])$ be any classical solution of (1.1), (1.2).

Then there exist families $\left\{f^{\varepsilon}\right\} \subseteq C^{0}([-1,1] \times[0, T])$ and $\left\{u^{\varepsilon}\right\} \subseteq C^{2,1}([-1,1] \times$ $[0, T])$ such that

$$
\begin{aligned}
u_{t}^{\varepsilon}=\varphi^{\prime \prime}\left(u_{x}^{\varepsilon}\right) u_{x x}^{\varepsilon}-\varepsilon u_{x x x x}^{\varepsilon}+f^{\varepsilon}(x, t) & \forall(x, t) \in[-1,1] \times[0, T], \\
u_{x}^{\varepsilon}(x, t)=u_{x x x}^{\varepsilon}(x, t)=0 & \forall(x, t) \in\{-1,1\} \times[0, T], \\
u^{\varepsilon} \rightarrow u & \text { in } C^{2,1}([-1,1] \times[0, T]), \\
f^{\varepsilon} \rightarrow 0 & \text { in } C^{0}([-1,1] \times[0, T]) .
\end{aligned}
$$

We chose fourth order regularizations only because in this framework the result can be stated without further definitions. An analogous statement holds true for higher order regularizations (for instance six order regularization) and for the semidiscrete scheme considered in [9].

\section{Proofs}

3.1. Compactness and comparison results. In this section we collect two technical results. They look classical, but we did not find these exact statements in the literature. For this reason in both cases we give a sketch of the proof for the convenience of the reader.

The first one is a compactness result. We recall that $C^{1 / 2,1 / 4}([a, b] \times[0, T])$ is the space of functions $f:[a, b] \times[0, T] \rightarrow \mathbb{R}$ for which there exists a constant $N$ such that

$$
\left|f\left(x_{1}, t_{1}\right)-f\left(x_{2}, t_{2}\right)\right| \leq N\left(\left|x_{1}-x_{2}\right|^{1 / 2}+\left|t_{1}-t_{2}\right|^{1 / 4}\right)
$$

for every $x_{1}, x_{2}$ in $[a, b]$ and every $t_{1}, t_{2}$ in $[0, T]$.

Lemma 3.1. For every $\varepsilon \in\left(0, \varepsilon_{0}\right)$ let $f^{\varepsilon} \in C^{1}([a, b] \times[0, T])$. Let us assume that there exists $M \in \mathbb{R}$ such that

$$
\begin{aligned}
\int_{0}^{T} \int_{a}^{b}\left[f_{t}^{\varepsilon}(x, t)\right]^{2} d x d t & \leq M & \forall \varepsilon \in\left(0, \varepsilon_{0}\right), \\
\int_{a}^{b}\left[f_{x}^{\varepsilon}(x, t)\right]^{2} d x & \leq M & \forall t \in[0, T], \forall \varepsilon \in\left(0, \varepsilon_{0}\right), \\
\left|f^{\varepsilon}(a, 0)\right| & \leq M & \forall \varepsilon \in\left(0, \varepsilon_{0}\right) .
\end{aligned}
$$

Then the family $\left\{f^{\varepsilon}\right\}$ is relatively compact in $C^{0}([a, b] \times[0, T])$ and any limit point belongs to $C^{1 / 2,1 / 4}([a, b] \times[0, T])$.

Proof. It is enough to prove that any function $f$ satisfying (3.2) and (3.3) also satisfies (3.1) with a constant $N$ independent of $\varepsilon$. At this point it is indeed easy to conclude that the family $\left\{f^{\varepsilon}\right\}$ is equicontinuous and equibounded; hence it is compact for the uniform convergence by the Ascoli-Arzela Theorem.

Moreover the rectangle $[a, b] \times[0, T]$ can be covered with a finite number $k$ of subrectangles $[a, b] \times\left[T_{i}, T_{i+1}\right]$ with $T_{i+1}-T_{i} \leq(b-a)^{2}$. If we prove that (3.1) holds true in each subrectangle with some constant $N$, then (3.1) holds true in the original rectangle with constant $k N$.

We can therefore assume, without loss of generality, that $\left|t_{1}-t_{2}\right|<(b-a)^{2}$. Now let us write

$$
\left|f\left(x_{1}, t_{1}\right)-f\left(x_{2}, t_{2}\right)\right| \leq\left|f\left(x_{1}, t_{1}\right)-f\left(x_{2}, t_{1}\right)\right|+\left|f\left(x_{2}, t_{1}\right)-f\left(x_{2}, t_{2}\right)\right|=: A+B .
$$


By (3.3) and Hölder's inequality we have that

$$
A \leq\left|\int_{x_{1}}^{x_{2}} f_{x}\left(x, t_{1}\right) d x\right| \leq M^{1 / 2}\left|x_{2}-x_{1}\right|^{1 / 2} .
$$

In order to estimate $B$, let us set $B_{\delta}\left(x_{2}\right)=\left\{x \in[a, b]:\left|x-x_{2}\right|<\delta\right\}$. If $\delta$ is smaller than $b-a$, then $\delta \leq\left|B_{\delta}\left(x_{2}\right)\right| \leq 2 \delta$. Moreover there exist $x_{\delta}$ and $y_{\delta}$ in $B_{\delta}\left(x_{2}\right)$ such that

$$
\frac{1}{\left|B_{\delta}\left(x_{2}\right)\right|} \int_{B_{\delta}\left(x_{2}\right)} f\left(x, t_{1}\right) d x=f\left(x_{\delta}, t_{1}\right), \quad \frac{1}{\left|B_{\delta}\left(x_{2}\right)\right|} \int_{B_{\delta}\left(x_{2}\right)} f\left(x, t_{2}\right) d x=f\left(y_{\delta}, t_{2}\right) .
$$

Now

$$
\begin{aligned}
B & \leq\left|f\left(x_{2}, t_{1}\right)-f\left(x_{\delta}, t_{1}\right)\right|+\left|f\left(x_{\delta}, t_{1}\right)-f\left(y_{\delta}, t_{2}\right)\right|+\left|f\left(y_{\delta}, t_{2}\right)-f\left(x_{2}, t_{2}\right)\right| \\
& =: B_{1}+B_{2}+B_{3} .
\end{aligned}
$$

Using (3.3) as we did in (3.5), it is easy to see that $B_{1} \leq M^{1 / 2} \delta^{1 / 2}$ and $B_{3} \leq$ $M^{1 / 2} \delta^{1 / 2}$.

Moreover, by (3.2) and Hölder's inequality applied in the rectangle $B_{\delta}\left(x_{2}\right) \times$ $\left[t_{1}, t_{2}\right]$ (we assume that $t_{1}<t_{2}$ without loss of generality) we have that

$$
\begin{aligned}
B_{2} & \leq \frac{1}{\left|B_{\delta}\left(x_{2}\right)\right|} \int_{B_{\delta}\left(x_{2}\right)}\left|f\left(x, t_{1}\right)-f\left(x, t_{2}\right)\right| d x \\
& \leq \frac{1}{\left|B_{\delta}\left(x_{2}\right)\right|} \int_{B_{\delta}\left(x_{2}\right)} \int_{t_{1}}^{t_{2}}\left|f_{t}(x, t)\right| d x d t \\
& \leq \frac{1}{\left|B_{\delta}\left(x_{2}\right)\right|}\left\{\left|B_{\delta}\left(x_{2}\right)\right| \cdot\left|t_{2}-t_{1}\right|\right\}^{1 / 2} M^{1 / 2} \\
& \leq M^{1 / 2} \delta^{-1 / 2}\left|t_{2}-t_{1}\right|^{1 / 2} .
\end{aligned}
$$

Putting it all together, we have that

$$
A+B \leq M^{1 / 2}\left|x_{2}-x_{1}\right|^{1 / 2}+2 M^{1 / 2} \delta^{1 / 2}+M^{1 / 2} \delta^{-1 / 2}\left|t_{2}-t_{1}\right|^{1 / 2},
$$

and therefore (3.1) follows by setting $\delta:=\left|t_{2}-t_{1}\right|^{1 / 2}$ (which we assumed to be smaller than $b-a)$.

The second result is one of the many variants of the comparison principle for fully nonlinear parabolic PDEs.

Lemma 3.2. Let $\Omega \subseteq \mathbb{R}^{2}$, and let $\psi:[a, b] \times[0, T] \times \Omega \times \mathbb{R} \rightarrow \mathbb{R}$ be a continuous function which is nondecreasing in the last variable, i.e.,

$$
\psi(x, t, p, q, r) \geq \psi(x, t, p, q, s) \quad \forall r \geq s
$$

for all admissible values of $x, t, p, q$ (this condition is usually called weak or degenerate ellipticity).

Let $u \in C^{0}([a, b] \times[0, T]) \cap C^{2,1}((a, b) \times(0, T])$ be a function such that

$$
\begin{array}{cc}
\left(u(x, t), u_{x}(x, t)\right) \in \Omega & \forall(x, t) \in(a, b) \times(0, T], \\
u_{t}=\psi\left(x, t, u, u_{x}, u_{x x}\right) & \text { in }(a, b) \times(0, T] .
\end{array}
$$

Let $v \in C^{0}([a, b] \times[0, T]) \cap C^{2,1}((a, b) \times(0, T])$, and let

$$
V_{\Omega}:=\left\{(x, t) \in(a, b) \times(0, T]:\left(v(x, t), v_{x}(x, t)\right) \in \Omega\right\} .
$$


Let us assume that

$$
\begin{aligned}
v_{t} & >\psi\left(x, t, v, v_{x}, v_{x x}\right) \quad \forall(x, t) \in V_{\Omega}, \\
v(x, t) & >u(x, t) \quad \forall(x, t) \in \partial_{P}([a, b] \times[0, T]) .
\end{aligned}
$$

Then $v(x, t)>u(x, t)$ for every $(x, t) \in[a, b] \times[0, T]$.

An analogous statement can be obtained by reversing the inequality signs in (3.9), (3.10), and in the conclusion.

Proof. Let $z(x, t):=v(x, t)-u(x, t)$. We claim that $z(x, t)>0$ for every $(x, t) \in$ $[a, b] \times[0, T]$. Let us assume by contradiction that this is not the case, and let

$$
t_{0}:=\inf \{t \in[0, T]: z(x, t) \leq 0 \text { for some } x \in[a, b]\} .
$$

Then it is easy to see that there exists $x_{0} \in[a, b]$ such that $z\left(x_{0}, t_{0}\right)=0$; hence $v\left(x_{0}, t_{0}\right)=u\left(x_{0}, t_{0}\right)=: p_{0}$. From (3.10) and the continuity of $z$ it follows that $t_{0}>0$ and $x_{0} \in(a, b)$. Therefore $z_{t}\left(x_{0}, t_{0}\right) \leq 0, z_{x}\left(x_{0}, t_{0}\right)=0$; hence $u_{x}\left(x_{0}, t_{0}\right)=$ $v_{x}\left(x_{0}, t_{0}\right)=: q_{0}$, and $z_{x x}\left(x_{0}, t_{0}\right) \geq 0$, and hence $v_{x x}\left(x_{0}, t_{0}\right) \geq u_{x x}\left(x_{0}, t_{0}\right)$.

In particular $\left(x_{0}, t_{0}\right) \in V_{\Omega}$; hence by (3.9), (3.8), and the weak ellipticity (3.6) of $\psi$ we have that

$$
\begin{aligned}
0 \geq z_{t}\left(x_{0}, t_{0}\right) & =v_{t}\left(x_{0}, t_{0}\right)-u_{t}\left(x_{0}, t_{0}\right) \\
& >\psi\left(x_{0}, t_{0}, p_{0}, q_{0}, v_{x x}\left(x_{0}, t_{0}\right)\right)-\psi\left(x_{0}, t_{0}, p_{0}, q_{0}, u_{x x}\left(x_{0}, t_{0}\right)\right) \geq 0,
\end{aligned}
$$

which is absurd.

\subsection{Proof of Theorem 2.3 .}

Global existence and maximum principle for $u_{x}$. In order to prove existence of a global solution of (2.16), (2.17), we take a function $\varphi_{\varepsilon} \in C^{\infty}(\mathbb{R})$ which coincides with $\varphi$ in the interval $\left(\alpha_{\varepsilon}, \beta_{\varepsilon}\right)$ and such that $\varphi_{\varepsilon}^{\prime \prime}(\sigma) \geq \nu>0$ for every $\sigma \in \mathbb{R}$. Problem (2.16), (2.17), with $\varphi_{\varepsilon}$ instead of $\varphi$, is strictly parabolic. By well knows results (see for example [14]) it admits a unique global solution with the regularity stated in (2.18).

Since the initial condition $u_{0}^{\varepsilon}$ satisfies (2.15), the classical maximum principle for $u_{x}^{\varepsilon}$ implies that this solution satisfies (2.19), and in particular it is also a solution of (2.16), (2.17) with the original $\varphi$. Finally, since the equation is strictly parabolic, (2.20) is equivalent to saying that $u_{t}^{\varepsilon}(x, t)=0$ for $x=a$ and $x=b$, which is a consequence of the Dirichlet boundary condition.

Global estimates on $u_{t}$. Computing the time derivative and integrating by parts, we have that

$$
\begin{aligned}
\frac{\mathrm{d}}{\mathrm{d} t}\left(\int_{a}^{b}\left[u_{t}^{\varepsilon}\right]^{2} d x\right) & =2 \int_{a}^{b} u_{t}^{\varepsilon}\left(\varphi^{\prime}\left(u_{x}^{\varepsilon}\right)\right)_{x t} d x \\
& =-2 \int_{a}^{b} u_{t x}^{\varepsilon}\left(\varphi^{\prime}\left(u_{x}^{\varepsilon}\right)\right)_{t} d x \\
& =-2 \int_{a}^{b} \varphi^{\prime \prime}\left(u_{x}^{\varepsilon}\right)\left[u_{t x}^{\varepsilon}\right]^{2} d x,
\end{aligned}
$$

where we neglected the boundary terms in the integration by parts because $u_{t}^{\varepsilon}$ is zero for $x \in\{a, b\}$ due to the Dirichlet boundary conditions coming from (2.17). 
Integrating in $[0, T]$, we obtain that

$$
\int_{a}^{b}\left[u_{t}^{\varepsilon}(x, T)\right]^{2} d x+2 \int_{0}^{T} \int_{a}^{b} \varphi^{\prime \prime}\left(u_{x}^{\varepsilon}\right)\left[u_{x t}^{\varepsilon}\right]^{2} d x d t \leq \int_{a}^{b}\left[u_{t}^{\varepsilon}(x, 0)\right]^{2} d x .
$$

Since the right hand side can be estimated independently of $\varepsilon$, inequalities (2.21) and (2.22) easily follow.

Uniform strict parabolicity in the interior. We have to prove that $u_{x}^{\varepsilon}(x, t)$ is bounded away from $\alpha$ and $\beta$. Without loss of generality let us concentrate on $\beta$ (the other case is quite similar).

If $\varphi^{\prime \prime}(\beta)>0$, then by (2.6) and (2.7) we have that $u_{0 x}(x)$ is bounded away from $\beta$ for every $x \in[a, b]$; hence also $u_{0 x}^{\varepsilon}(x)$ is bounded away from $\beta$ independently of $\varepsilon$. By the maximum principle $u_{x}^{\varepsilon}(x, t)$ has the same bound for every $(x, t) \in$ $[a, b] \times[0,+\infty)$.

Let us assume now that $\varphi^{\prime \prime}(\beta)=0$. We consider the function

$$
z_{0}(x):=\frac{(x-a)^{2}(b-x)^{2}\left(\beta-u_{0 x}(x)\right)}{2(b-a)^{4}}
$$

we choose

$$
k>\sup _{\sigma \in(\alpha, \beta)} \frac{\varphi^{\prime \prime}(\sigma)}{\beta-\sigma} \cdot \max _{x \in[a, b]}\left|z_{0 x x}(x)\right|+\max _{\sigma \in(\alpha, \beta)}\left|\varphi^{\prime \prime \prime}(\sigma)\right| \cdot \sup _{x \in(a, b)} \frac{z_{0 x}^{2}(x)}{z_{0}(x)}
$$

(the first supremum is finite because $\varphi^{\prime \prime}(\beta)=0$, and the second supremum is finite because $\left.z_{0 x}(a)=z_{0 x}(b)=0\right)$, and we claim that

$$
u_{x}^{\varepsilon}(x, t)<\beta-\frac{z_{0}(x)}{1+k t} \quad \forall(x, t) \in[a, b] \times[0,+\infty) .
$$

Since $z_{0}(x)>0$ for every $x \in(a, b)$, this proves that $u_{x}^{\varepsilon}$ is bounded away from $\beta$, independently of $\varepsilon$, in every rectangle $\left[x_{1}, x_{2}\right] \times[0, T]$ with $\left[x_{1}, x_{2}\right] \subseteq(a, b)$.

In order to prove (3.11), we set for simplicity $v:=u_{x}^{\varepsilon}$, we denote by $z(x, t)$ the right hand side, and we apply Lemma 3.2 to the functions $v$ and $z$. Indeed a simple calculation shows that $v$ satisfies

$$
v_{t}=\varphi^{\prime \prime}(v) v_{x x}+\varphi^{\prime \prime \prime}(v) v_{x}^{2} \quad \text { in }[a, b] \times[0,+\infty) .
$$

The right hand side of (3.12) satisfies the weak ellipticity condition (3.6) with $\Omega=(\alpha, \beta) \times \mathbb{R}$. Moreover for every $(x, t) \in(a, b) \times[0,+\infty)$ it is easy to see that $\alpha<z(x, t)<\beta$; hence by definition of $z$ and $k$ we have that

$$
\begin{aligned}
\varphi^{\prime \prime}(z) z_{x x}+\varphi^{\prime \prime \prime}(z) z_{x}^{2} & =-\varphi^{\prime \prime}(z) \frac{z_{0 x x}}{1+k t}+\varphi^{\prime \prime \prime}(z) \frac{z_{0 x}^{2}}{(1+k t)^{2}} \\
& =-\frac{\varphi^{\prime \prime}(z)}{\beta-z} \frac{z_{0}}{(1+k t)^{2}} z_{0 x x}+\varphi^{\prime \prime \prime}(z) \frac{z_{0}}{(1+k t)^{2}} \frac{z_{0 x}^{2}}{z_{0}} \\
& \leq \frac{z_{0}}{(1+k t)^{2}}\left\{\frac{\varphi^{\prime \prime}(z)}{\beta-z}\left|z_{0 x x}\right|+\left|\varphi^{\prime \prime \prime}(z)\right| \frac{z_{0 x}^{2}}{z_{0}}\right\} \\
& <k \frac{z_{0}}{(1+k t)^{2}}=z_{t},
\end{aligned}
$$

which proves that $z$ is a supersolution of (3.12). It remains to show that $z(x, t)>$ $v(x, t)$ on the parabolic boundary of $[a, b] \times[0,+\infty)$. For $x=a$ and $x=b$ we have 
that $z(x, t)=\beta>\beta_{\varepsilon} \geq v(x, t)$, while for $t=0$ we have that

$$
z(x, 0) \geq \beta-\frac{\beta-u_{0 x}(x)}{2}=\frac{u_{0 x}(x)+\beta}{2}>\frac{u_{0 x}(x)}{1+\varepsilon}+\frac{\varepsilon(\alpha+\beta)}{2(1+\varepsilon)}=v(x, 0),
$$

where the last inequality can be proved simply by checking that it holds true for $u_{0 x}=\alpha$ and $u_{0 x}=\beta$ and that both sides are affine with respect to $u_{0 x}$. This completes the proof of (2.23).

Since $u_{x}^{\varepsilon}$ is bounded away from the possible zeroes of $\varphi^{\prime \prime}$, estimate (2.24) easily follows.

Interior estimates. Let $y_{1}:=\left(x_{1}+a\right) / 2, y_{2}:=\left(x_{2}+b\right) / 2$. Let $r \in C^{\infty}(\mathbb{R})$ be a cut-off function such that $r(x)=1$ for $x \in\left[x_{1}, x_{2}\right], r(x)=0$ for $x \leq y_{1}$ or $x \geq y_{2}$, and $0<r(x)<1$ otherwise. Let us set for simplicity

$$
\begin{aligned}
E(t) & :=\int_{a}^{b} r^{2}\left[u_{x t}^{\varepsilon}\right]^{2} d x, & F(t) & :=\int_{a}^{b} r^{2} \varphi^{\prime \prime}\left(u_{x}^{\varepsilon}\right)\left[u_{x x t}^{\varepsilon}\right]^{2} d x, \\
G(t) & :=\int_{a}^{b} \varphi^{\prime \prime}\left(u_{x}^{\varepsilon}\right)\left[u_{x t}^{\varepsilon}\right]^{2} d x, & H(t) & :=\max _{x \in[a, b]}\left|r(x) u_{x x}^{\varepsilon}(x, t)\right| .
\end{aligned}
$$

In the following estimates we introduce constants $c_{1}, \ldots, c_{14}$, all independent of $\varepsilon$.

Thanks to (2.24) the function $\varphi^{\prime \prime}\left(u_{x}^{\varepsilon}\right)$ is bounded away from 0 in the rectangle $\left[x_{1}, x_{2}\right] \times[0, T] ;$ hence

$$
\int_{y_{1}}^{y_{2}}\left[u_{x t}^{\varepsilon}\right]^{2} d x \leq c_{1} \int_{y_{1}}^{y_{2}} \varphi^{\prime \prime}\left(u_{x}^{\varepsilon}\right)\left[u_{x t}^{\varepsilon}\right]^{2} d x \leq c_{1} G(t) .
$$

Taking the time derivative and integrating by parts, we have that

$$
\begin{aligned}
E^{\prime}(t)= & 2 \int_{a}^{b} r^{2} u_{x t}^{\varepsilon} u_{x t t}^{\varepsilon} d x \\
= & -2 \int_{a}^{b}\left(r^{2} u_{x t}^{\varepsilon}\right)_{x} u_{t t}^{\varepsilon} d x \\
= & -2 \int_{a}^{b}\left(2 r r_{x} u_{x t}^{\varepsilon}+r^{2} u_{x x t}^{\varepsilon}\right)\left(\varphi^{\prime \prime}\left(u_{x}^{\varepsilon}\right) u_{x t}^{\varepsilon}\right)_{x} d x \\
= & -4 \int_{a}^{b} r r_{x}\left[u_{x t}^{\varepsilon}\right]^{2} \varphi^{\prime \prime \prime}\left(u_{x}^{\varepsilon}\right) u_{x x}^{\varepsilon} d x-4 \int_{a}^{b} r r_{x} u_{x t}^{\varepsilon} \varphi^{\prime \prime}\left(u_{x}^{\varepsilon}\right) u_{x x t}^{\varepsilon} d x \\
& -2 \int_{a}^{b} r^{2} \varphi^{\prime \prime \prime}\left(u_{x}^{\varepsilon}\right) u_{x x}^{\varepsilon} u_{x t}^{\varepsilon} u_{x x t}^{\varepsilon} d x-2 \int_{a}^{b} r^{2} \varphi^{\prime \prime}\left(u_{x}^{\varepsilon}\right)\left[u_{x x t}^{\varepsilon}\right]^{2} d x \\
=: & I_{1}(t)+I_{2}(t)+I_{3}(t)+I_{4}(t) .
\end{aligned}
$$

Now we estimate separately the four terms. First of all

$$
I_{1}(t) \leq c_{2} H(t) \int_{y_{1}}^{y_{2}}\left[u_{x t}^{\varepsilon}\right]^{2} d x \leq c_{3}\left(1+H^{2}(t)\right) G(t)
$$


Using the well known inequality $2 A B \leq \eta A^{2}+\eta^{-1} B^{2}$ with suitable choices of $A, B, \eta$, we have that

$$
\begin{aligned}
I_{2}(t) & =-4 \int_{a}^{b}\left(r \sqrt{\varphi^{\prime \prime}\left(u_{x}^{\varepsilon}\right)} u_{x x t}^{\varepsilon}\right)\left(r_{x} \sqrt{\varphi^{\prime \prime}\left(u_{x}^{\varepsilon}\right)} u_{x t}^{\varepsilon}\right) d x \\
& \leq \frac{1}{2} \int_{a}^{b} r^{2} \varphi^{\prime \prime}\left(u_{x}^{\varepsilon}\right)\left[u_{x x t}^{\varepsilon}\right]^{2} d x+c_{4} \int_{a}^{b} r_{x}^{2} \varphi^{\prime \prime}\left(u_{x}^{\varepsilon}\right)\left[u_{x t}^{\varepsilon}\right]^{2} d x \\
& \leq \frac{1}{2} F(t)+c_{5} G(t),
\end{aligned}
$$

and

$$
\begin{aligned}
I_{3}(t) & =-2 \int_{y_{1}}^{y_{2}}\left(r \sqrt{\varphi^{\prime \prime}\left(u_{x}^{\varepsilon}\right)} u_{x x t}^{\varepsilon}\right)\left(r \frac{\varphi^{\prime \prime \prime}\left(u_{x}^{\varepsilon}\right)}{\sqrt{\varphi^{\prime \prime}\left(u_{x}^{\varepsilon}\right)}} u_{x x}^{\varepsilon} u_{x t}^{\varepsilon}\right) d x \\
& \leq \frac{1}{2} \int_{y_{1}}^{y_{2}} r^{2} \varphi^{\prime \prime}\left(u_{x}^{\varepsilon}\right)\left[u_{x x t}^{\varepsilon}\right]^{2} d x+c_{6} \int_{y_{1}}^{y_{2}} r^{2} \frac{\left[\varphi^{\prime \prime \prime}\left(u_{x}^{\varepsilon}\right)\right]^{2}}{\varphi^{\prime \prime}\left(u_{x}^{\varepsilon}\right)}\left[u_{x x}^{\varepsilon}\right]^{2}\left[u_{x t}^{\varepsilon}\right]^{2} d x \\
& \leq \frac{1}{2} F(t)+c_{7} H^{2}(t) G(t) .
\end{aligned}
$$

Since $I_{4}(t)=-2 F(t)$, putting it all together, we obtain that

$$
E^{\prime}(t) \leq-F(t)+c_{8} G(t)\left\{1+H^{2}(t)\right\} .
$$

Now we need an estimate for $H^{2}(t)$. Using (2.24) once more, for every $s \in\left[y_{1}, y_{2}\right]$ we have that

$$
\begin{aligned}
\left|r(s) u_{x x}^{\varepsilon}(s, t)\right|^{2} & \leq c_{9}\left|r(s) \varphi^{\prime \prime}\left(u_{x}^{\varepsilon}(s, t)\right) u_{x x}^{\varepsilon}(s, t)\right|^{2} \\
& =c_{9}\left|r(s) u_{t}^{\varepsilon}(s, t)\right|^{2} \\
& =c_{9}\left[\int_{y_{1}}^{s}\left(r u_{t}^{\varepsilon}\right)_{x} d x\right]^{2} \\
& \leq c_{9}\left(s-y_{1}\right) \int_{y_{1}}^{s}\left[\left(r u_{t}^{\varepsilon}\right)_{x}\right]^{2} d x \\
& \leq c_{9}(b-a) \int_{a}^{b}\left[r_{x} u_{t}^{\varepsilon}+r u_{t x}^{\varepsilon}\right]^{2} d x \\
& \leq c_{10} \int_{a}^{b}\left[u_{t}^{\varepsilon}\right]^{2} d x+c_{11} E(t) .
\end{aligned}
$$

By (2.21) the first summand in the last line is bounded independently of $\varepsilon$ and $t$. We have thus proved that

$$
H^{2}(t) \leq c_{12}+c_{11} E(t) \quad \forall t \in[0, T],
$$

so that, coming back to (3.15), we have that

$$
E^{\prime}(t) \leq c_{13} G(t) E(t)+c_{14} G(t)-F(t) .
$$

Let $\Gamma(t)$ be the function such that $\Gamma^{\prime}(t)=c_{13} G(t)$ and $\Gamma(0)=0$. By the usual comparison argument for ODEs we obtain that

$$
E(t)+e^{\Gamma(t)} \int_{0}^{t} e^{-\Gamma(s)} F(s) d s \leq E(0) e^{\Gamma(t)}+c_{14} e^{\Gamma(t)} \int_{0}^{t} e^{-\Gamma(s)} G(s) d s
$$


for every $t \in[0, T]$. By (2.22) the integral of $G$, hence also $\Gamma(t)$, is bounded from above independently of $\varepsilon$. Therefore the right hand side of (3.17) is bounded independently of $\varepsilon$.

Since $r(x)=1$ in $\left[x_{1}, x_{2}\right]$, this proves (2.25) and (2.26) by definition of $E(t)$ and $F(t)$. Analogously, by definition of $H(t),(2.27)$ follows from (3.16) and the uniform bound for the right hand side of (3.17).

Uniform vanishing of $u_{x x}^{\varepsilon}$ at the boundary. Let us prove first that there exist constants $c_{1}$ and $T_{1}>0$ such that

$$
u_{x x}^{\varepsilon}(x, t) \leq c_{1}(x-a) \quad \forall(x, t) \in[a, b] \times\left[0, T_{1}\right]
$$

To this end let us choose

$$
k_{1}>\max _{\sigma \in[\alpha, \beta]}\left|\varphi^{\prime \prime \prime}(\sigma)\right|, \quad k_{2}>\max _{\sigma \in[\alpha, \beta]}\left|\varphi^{I V}(\sigma)\right|, \quad k_{3}>\sup _{x \in(a, b]} \frac{\left|u_{0 x x}(x)\right|}{x-a}
$$

(the supremum is finite because $u_{0 x x}(a)=0$ ), let $g(t)$ be the solution of the Cauchy problem

$$
g^{\prime}(t)=3 k_{1} g^{2}(t)+4 k_{2}(b-a)^{2} g^{3}(t) \quad g(0)=k_{3},
$$

let $h(t)$ be the solution of the Cauchy problem

$$
h^{\prime}(t)=3 k_{1} g(t) h(t)+4 k_{2} h^{3}(t) \quad h(0)=1,
$$

and let $T_{1}>0$ be such that $g(t)$ and $h(t)$ are defined at least for $t \in\left[0, T_{1}\right]$.

If we prove that for every $\eta \in(0,1)$ we have that

$$
u_{x x}^{\varepsilon}(x, t)<g(t)(x-a)+\eta h(t) \quad \forall(x, t) \in[a, b] \times\left[0, T_{1}\right],
$$

then 3.18 with $c_{1}:=g\left(T_{1}\right)$ follows by letting $\eta \rightarrow 0^{+}$.

In order to prove (3.19), for simplicity we set $w^{\varepsilon}=u_{x x}^{\varepsilon}$, we denote the right hand side by $z(x, t)$, and we apply Lemma 3.2 to the functions $w^{\varepsilon}$ and $z$. Indeed with some calculations it turns out that

$$
\begin{aligned}
w_{t}^{\varepsilon} & =\varphi^{\prime \prime}\left(u_{x}^{\varepsilon}\right) w_{x x}^{\varepsilon}+3 \varphi^{\prime \prime \prime}\left(u_{x}^{\varepsilon}\right) w^{\varepsilon} w_{x}^{\varepsilon}+\varphi^{I V}\left(u_{x}^{\varepsilon}\right)\left[w^{\varepsilon}\right]^{3} \\
& =: a^{\varepsilon}(x, t) w_{x x}^{\varepsilon}+b^{\varepsilon}(x, t) w^{\varepsilon} w_{x}^{\varepsilon}+c^{\varepsilon}(x, t)\left[w^{\varepsilon}\right]^{3},
\end{aligned}
$$

where (3.20) satisfies the weak ellipticity condition (3.6) with $\Omega=\mathbb{R} \times \mathbb{R}$.

Moreover

$$
\begin{aligned}
a^{\varepsilon} z_{x x} & +b^{\varepsilon} z z_{x}+c^{\varepsilon} z^{3}=b^{\varepsilon}(g(t)(x-a)+\eta h(t)) g(t)+c^{\varepsilon}(g(t)(x-a)+\eta h(t))^{3} \\
\leq & b^{\varepsilon}(x-a) g^{2}(t)+\eta b^{\varepsilon} h(t) g(t)+4 c^{\varepsilon} g^{3}(t)(x-a)^{3}+4 c^{\varepsilon} \eta^{3} h^{3}(t) \\
< & (x-a)\left\{3 k_{1} g^{2}(t)+4 k_{2}(b-a)^{2} g^{3}(t)\right\} \\
& \quad+\eta\left\{3 k_{1} g(t) h(t)+4 k_{2} h^{3}(t)\right\} \\
= & (x-a) g^{\prime}(t)+\eta h^{\prime}(t)=z_{t}
\end{aligned}
$$

which proves that $z$ is a supersolution of the same equation. It remains to show that $z(x, t)>w^{\varepsilon}(x, t)$ on the parabolic boundary of $[a, b] \times\left[0, T_{1}\right]$. This is true because for $x=a$ and $x=b$ we have that $z$ is positive and $w^{\varepsilon}$ is zero by (2.20), while for $t=0$ we have that

$$
z(x, 0)=k_{3}(x-a)+\eta>\left|u_{0 x x}(x)\right| \geq u_{0 x x}^{\varepsilon}(x)=w^{\varepsilon}(x, 0) .
$$

Therefore (3.19) follows from Lemma 3.2, and this completes the proof of (3.18). 
With similar arguments we can prove that there exist constants $c_{2}, c_{3}, c_{4}$, and positive times $T_{2}, T_{3}, T_{4}$ such that

$$
\begin{array}{ll}
u_{x x}^{\varepsilon}(x, t) \geq-c_{2}(x-a) & \forall(x, t) \in[a, b] \times\left[0, T_{2}\right], \\
u_{x x}^{\varepsilon}(x, t) \leq c_{3}(b-x) & \forall(x, t) \in[a, b] \times\left[0, T_{3}\right], \\
u_{x x}^{\varepsilon}(x, t) \geq-c_{4}(b-x) & \forall(x, t) \in[a, b] \times\left[0, T_{4}\right] .
\end{array}
$$

In conclusion, from inequalities (3.18), (3.21), (3.22), (3.23) we easily obtain (2.28) for $T_{0}:=\min \left\{T_{1}, T_{2}, T_{3}, T_{4}\right\}$.

\subsection{Proof of Theorem 2.2 .}

Uniqueness. Under condition (2.11) equation (2.9) is weakly parabolic. Since $u$ is prescribed on the parabolic boundary, uniqueness follows from standard techniques, for example an estimate of the $L^{2}$ norm of the difference of two solutions, or comparison arguments such as Lemma 3.2

Convergence. We show that there exists $u \in C^{0}([a, b] \times[0,+\infty)) \cap C^{\infty}((a, b) \times$ $[0,+\infty))$ satisfying (2.9), (2.10), (2.11) and such that

$$
\begin{aligned}
u^{\varepsilon} \rightarrow u & \text { uniformly on compact subsets of }[a, b] \times[0+\infty), \\
u_{x}^{\varepsilon} \rightarrow u_{x} & \text { uniformly on compact subsets of }(a, b) \times[0+\infty), \\
u_{t}^{\varepsilon} \rightarrow u_{t} & \text { uniformly on compact subsets of }(a, b) \times[0+\infty), \\
u_{x x}^{\varepsilon} \rightarrow u_{x x} & \text { uniformly on compact subsets of }(a, b) \times[0+\infty) .
\end{aligned}
$$

First of all, since (2.9), (2.10), (2.11) uniquely characterize the possible limits, it is enough to prove convergence up to subsequences.

In order to prove (3.24), we apply Lemma 3.1 to the family $\left\{u^{\varepsilon}\right\}$ in any rectangle $[a, b] \times[0, T]$. Assumptions (3.2) and (3.3) follow from (2.21) and (2.19), while (3.4) is a direct consequence of the initial condition. This also proves that $u$ satisfies the initial and boundary conditions (2.10).

In order to prove that $u_{x}$ exists and satisfies (3.25), we apply Lemma 3.1 to the family $\left\{u_{x}^{\varepsilon}\right\}$ in any rectangle $\left[x_{1}, x_{2}\right] \times[0, T]$ with $a<x_{1}<x_{2}<b$. The estimate on $u_{x t}^{\varepsilon}$ required in (3.2) follows from (2.25); the estimate on $u_{x x}^{\varepsilon}$ required in (3.3) is a consequence of (2.27); finally (3.4) follows from (2.19).

In order to prove that $u_{t}$ exists and satisfies (3.26), we apply Lemma 3.1 to the family $\left\{u_{t}^{\varepsilon}\right\}$ in any rectangle $\left[x_{1}, x_{2}\right] \times[0, T]$ with $a<x_{1}<x_{2}<b$. The estimate on $u_{t x}^{\varepsilon}$ required in (3.3) is (2.25). Moreover, since

$$
u_{t t}^{\varepsilon}=\varphi^{\prime \prime \prime}\left(u_{x}^{\varepsilon}\right) u_{x x}^{\varepsilon} u_{x t}^{\varepsilon}+\varphi^{\prime \prime}\left(u_{x}^{\varepsilon}\right) u_{x x t}^{\varepsilon},
$$

estimate (3.2) follows from (2.27), (2.25), and (2.26). Finally, (3.4) trivially follows from the fact that $u_{t}^{\varepsilon}$ is determined at $t=0$ by the initial condition.

In order to prove that $u_{x x}$ exists and satisfies (3.27), we recall that

$$
u_{x x}^{\varepsilon}=\frac{u_{t}^{\varepsilon}}{\varphi^{\prime \prime}\left(u_{x}^{\varepsilon}\right)} \text {. }
$$

Since the denominator is bounded away from zero because of (2.24), the uniform convergence of $u_{x x}^{\varepsilon}$ follows from the uniform convergence of $u_{t}^{\varepsilon}$ and $u_{x}^{\varepsilon}$. This also proves that $u$ is a solution of (2.9) in $(a, b) \times[0,+\infty)$.

Passing (2.23) to the limit, we deduce (2.11). As a consequence, the equation is strictly parabolic in any compact subset of $(a, b) \times[0,+\infty)$; hence $u$ is automatically of class $C^{\infty}$ in this set. 
Existence of $u_{x}$ at the boundary. We prove that there exists $T_{0}>0$ such that $u_{x}(x, t)$ exists for $(x, t) \in\{a, b\} \times\left[0, T_{0}\right]$ and satisfies (2.12).

Let us concentrate first on the endpoint $x=a$. By our assumptions on $\varphi$ and $u_{0}$ we have that $u_{0 x}(a) \in\{\alpha, \beta\}$. Let us assume that $u_{0 x}(a)=\alpha$; hence $\varphi^{\prime \prime}(\alpha)=0$.

Now we choose positive numbers $k, h, T_{0}$ such that

$$
k>18 \sup _{\sigma \in(\alpha, \beta)} \frac{\varphi^{\prime \prime}(\sigma)}{\sigma-\alpha}, \quad \frac{1}{h}>\sup _{x \in(a, b)}\left|\frac{u_{0}(x)-u_{0}(a)-\alpha(x-a)}{(x-a)^{3}}\right|, \quad T_{0}<\frac{h}{k}
$$

(both suprema are finite because $\varphi^{\prime \prime}(\alpha)=0$ and $u_{0 x x}(a)=0$ ), and we claim that

$$
u_{0}(a)+\alpha(x-a) \leq u(x, t) \leq u_{0}(a)+\alpha(x-a)+\frac{(x-a)^{3}}{h-k t}
$$

for every $(x, t) \in[a, b] \times\left[0, T_{0}\right]$. Since the left and the right hand sides have the same value at $x=a$ and since they both have derivative equal to $\alpha$ at $x=a$, it follows that $u_{x}(a, t)$ exists and is equal to $\alpha$ for every $t \in\left[0, T_{0}\right]$.

So we have only to prove (3.28). The inequality on the left is true for every $t \geq 0$ because of (2.11). Let us denote by $v(x, t)$ the right hand side of (3.28). The inequality on the right is equivalent to showing that $v(x, t)+\eta>u(x, t)$ for every $\eta>0$ and every $(x, t) \in[a, b] \times\left[0, T_{0}\right]$.

To this end we want to apply Lemma 3.2 to the functions $u$ and $v+\eta$. The function $u$ is a solution of equation (2.9), whose right hand side satisfies the weak ellipticity condition (3.6) with $\Omega=\mathbb{R} \times(\alpha, \beta)$. So we need only to verify that $v(x, t)+\eta>u(x, t)$ on the parabolic boundary of the rectangle and $v_{t}>\varphi^{\prime \prime}\left(v_{x}\right) v_{x x}$ when $\alpha<v_{x}<\beta$.

Inequality $v(x, t)+\eta>u(x, t)$ for $t=0$ is substantially equivalent to our choice of $h$, while for $x \in\{a, b\}$ we have that $v(x, t)+\eta>v(x, 0) \geq u(x, 0)=u(x, t)$. Finally, for simplicity setting $g(t)=(h-k t)^{-1}$ when $\alpha<v_{x}(x, t)=\alpha+3 g(t)(x-a)^{2}<\beta$, we have that

$$
\begin{aligned}
\varphi^{\prime \prime}\left(v_{x}\right) v_{x x} & =\varphi^{\prime \prime}\left(\alpha+3 g(t)(x-a)^{2}\right) \cdot 6 g(t)(x-a) \\
& =\frac{\varphi^{\prime \prime}\left(\alpha+3 g(t)(x-a)^{2}\right)}{3 g(t)(x-a)^{2}} \cdot 18 g^{2}(t)(x-a)^{3} \\
& <k g^{2}(t)(x-a)^{3} \\
& =g^{\prime}(t)(x-a)^{3}=v_{t} .
\end{aligned}
$$

This completes the proof when $u_{0 x}(a)=\alpha$.

If $u_{0 x}(a)=\beta$, then in a similar way we prove that

$$
u_{0}(a)+\beta(x-a) \geq u(x, t) \geq u_{0}(a)+\beta(x-a)-\frac{(x-a)^{3}}{h-k t},
$$

where now of course $\beta$ replaces $\alpha$ in the definitions of $h$ and $k$, and this is enough to conclude that $u_{x}(a, t)=\beta$ for every $x \in\left[0, T_{0}\right]$.

Analogous arguments work at the endpoint $x=b$.

Existence and continuity of $u_{x}, u_{t}, u_{x x}$ up to the boundary. Let $T_{0}>0$ be small enough so that (2.12) and estimate (2.28) hold true. We claim that for this choice of $T_{0}$ we have that $u$ belongs to $C^{2,1}\left([a, b] \times\left[0, T_{0}\right]\right)$ and satisfies (2.13).

Let us consider $u_{x}$. We have just shown that $u_{x}$ is defined for every $(x, t) \in$ $[a, b] \times\left[0, T_{0}\right]$. Moreover from (2.28) we obtain a uniform bound on $u_{x x}^{\varepsilon}$, which 
proves in particular that the functions $u_{x}^{\varepsilon}$ are Lipschitz continuous with respect to the $x$ variable in $[a, b] \times\left[0, T_{0}\right]$, with Lipschitz constant independent of $t$ and $\varepsilon$. In particular the limit $u_{x}$ turns out to be Lipschitz continuous with respect to the $x$ variable in $[a, b] \times\left[0, T_{0}\right]$, continuous with respect to both variables in $(a, b) \times\left[0, T_{0}\right]$, and constant for $x=a$ and $x=b$. This is enough to conclude that $u_{x}$ is continuous (with respect to both variables) in $[a, b] \times\left[0, T_{0}\right]$.

Now let us consider $u_{t}$, which coincides with $\varphi^{\prime \prime}\left(u_{x}\right) u_{x x}$ in $(a, b) \times\left[0, T_{0}\right]$. It is the product of the bounded function $u_{x x}$ and a function $\varphi^{\prime \prime}\left(u_{x}\right)$ which tends to 0 for $x \rightarrow a^{+}$and for $x \rightarrow b^{-}$. Therefore $u_{t}$ can be extended to $[a, b] \times\left[0, T_{0}\right]$ just by defining it to be 0 at $x=a$ or $x=b$.

Finally, we consider $u_{x x}$. Inequality (2.28) shows that the functions $u_{x x}^{\varepsilon}$ uniformly vanish at $x=a$ and $x=b$. Together with (3.27) this implies that the family $\left\{u_{x x}^{\varepsilon}\right\}$ uniformly converges in the whole rectangle $[a, b] \times\left[0, T_{0}\right]$. Moreover the limit is clearly $u_{x x}$ and vanishes at the endpoints, which proves (2.13).

Of course convergencies (3.25), (3.26), (3.27) are now uniform in $[a, b] \times\left[0, T_{0}\right]$.

3.4. Proof of Remark 2.4. Let $\left\{\rho_{\eta}(x)\right\}_{\eta \in(0,1)}$ be a family of mollifiers in the $x$ variable, which we assume to be even functions. Let us extend $u(x, t)$ to $[-2,2] \times$ $[0, T]$ in such a way that $u(1+x)=u(1-x)$ and $u(-1+x)=u(-1-x)$ for every $x \in(0,1)$. Let us consider $u^{\eta}(x, t):=u(x, t) * \rho_{\eta}(x)$ (convolution in the $x$ variable), defined for $x$ in a neighborhood of $[-1,1]$ and $t \in[0, T]$.

Since $u$ is of class $C^{2,1}$, we have that $u^{\eta} \rightarrow u, u_{t}^{\eta} \rightarrow u_{t}, u_{x}^{\eta} \rightarrow u_{x}, u_{x x}^{\eta} \rightarrow u_{x x}$ uniformly in $[-1,1] \times[0, T]$ as $\eta \rightarrow 0^{+}$.

Moreover from the symmetry properties of the extension of $u$ and of the mollifiers it follows that $u_{x}^{\eta}(x, t)=u_{x x x}^{\eta}(x, t)=0$ for $x=-1$ and $x=1$ (and the same for all derivatives with odd order). If we set

$$
g^{\varepsilon, \eta}:=u_{t}^{\eta}-\varphi^{\prime \prime}\left(u_{x}^{\eta}\right) u_{x x}^{\eta}+\varepsilon u_{x x x x}^{\eta}
$$

then we have that

$$
\lim _{\eta \rightarrow 0^{+}}\left(\lim _{\varepsilon \rightarrow 0^{+}} g^{\varepsilon, \eta}(x, t)\right)=0 \quad \text { uniformly in }[-1,1] \times[0, T] .
$$

By a well known property of double limits there exists a function $\eta(\varepsilon)$ such that $\eta(\varepsilon) \rightarrow 0^{+}$as $\varepsilon \rightarrow 0^{+}$and

$$
\lim _{\varepsilon \rightarrow 0^{+}} g^{\varepsilon, \eta(\varepsilon)}(x, t)=0 \quad \text { uniformly in }[-1,1] \times[0, T] .
$$

Therefore $u^{\varepsilon}:=u^{\eta(\varepsilon)}$ and $f^{\varepsilon}:=g^{\varepsilon, \eta(\varepsilon)}$ satisfy (2.29), (2.30), (2.31), and (2.32).

\section{REFERENCES}

[1] D. G. Aronson; Regularity properties of flows through porous media, SIAM J. Appl. Math. 17 (1969), 461-467. MR0247303 (40:571)

[2] D. G. Aronson, L. A. Caffarelli; Optimal regularity for one-dimensional porous medium flow, Rev. Mat. Iberoamericana 2 (1986), 357-366. MR913692 (88k:35219)

[3] G. Bellettini, G. Fusco; The $\Gamma$-limit and the related gradient flow for singular perturbation functionals of Perona-Malik type, Trans. Amer. Math. Soc. 360 (2008), 4929-4987. MR 2403710

[4] G. Bellettini, M. Novaga, E. Paolini; Global solutions to the gradient flow equation of a nonconvex functional, SIAM J. Math. Anal. 37 (2006), 1657-1687. MR2215602 (2007a:35060)

[5] Y. Chen, K. Zhang; Young measure solutions of the two-dimensional Perona-Malik equation in image processing, Commun. Pure Appl. Anal. 5 (2006), 615-635. MR2217594|(2006m:35145) 
[6] E. De Giorgi; Su alcuni problemi instabili legati alla teoria della visione, in Atti del convegno in onore di Carlo Ciliberto (Napoli, 1995), T. Bruno, P. Buonocore, L. Carbone, V. Esposito, eds., La Città del Sole, Napoli (1997), 91-98.

[7] S. Esedoglu; An analysis of the Perona-Malik scheme, Comm. Pure Appl. Math. 54 (2001), 1442-1487. MR1852979(2003i:94005)

[8] S. Esedoglu; Stability properties of the Perona-Malik scheme, SIAM J. Numer. Anal. 44 (2006), 1297-1313. MR2231865 (2007g:65081)

[9] M. Ghisi, M. Gobbino; Gradient estimates for the Perona-Malik equation. Math. Ann. 337 (2007), no. 3, 557-590. MR2274543 (2008b:35132)

[10] M. Gobbino; Entire solutions of the one-dimensional Perona-Malik equation. Comm. Partial Differential Equations 32 (2007), no. 4-6, 719-743. MR2334830(2008c:35123)

[11] K. Höllig; Existence of infinitely many solutions for a forward-backward heat equation, Trans. Amer. Math. Soc. 278 (1983), 299-316. MR697076 (84m:35062)

[12] B. Kawohl, N. Kutev; Maximum and comparison principle for one-dimensional anisotropic diffusion, Math. Ann. 311 (1998), 107-123. MR.1624275 (99h:35110)

[13] S. Kichenassamy; The Perona-Malik paradox, SIAM J. Appl. Math. 57 (1997), 1328-1342. MR.1470926 (98i:35088)

[14] O. A. Ladyženskaja, V. A. Solonnikov, N. N. Ural'ceva; Linear and quasilinear equations of parabolic type, Translations of Mathematical Monographs, Vol. 23, American Mathematical Society, Providence, R.I., 1967. MR0241822(39:3159b)

[15] P. Perona, J. Malik; Scale space and edge detection using anisotropic diffusion, IEEE Trans. Pattern Anal. Mach. Intell. 12 (1990), 629-639.

[16] S. Taheri, Q. Tang, K. Zhang; Young measure solutions and instability of the one-dimensional Perona-Malik equation, J. Math. Anal. Appl. 308 (2005), 467-490. MR2150102 (2007j:35095)

[17] K. Zhang; Existence of infinitely many solutions for the one-dimensional Perona-Malik problem, Calc. Var. 26 (2006), 171-199. MR2222243 (2007f:35153)

Dipartimento di Matematica "Leonida Tonelli", Università degli Studi di Pisa, Largo Bruno Pontecorvo 5, 56127 Pisa, Italy

E-mail address: ghisi@dm.unipi.it

Dipartimento di Matematica Applicata "Ulisse Dini", Università degli Studi di Pisa, Via Filippo Buonarroti 1C, 56127 Pisa, Italy

E-mail address: m.gobbino@dma.unipi.it 